\title{
Synaptic Correlates of Binocular Convergence: Just a Coincidence?
}

\author{
Benjamin Scholl ${ }^{1}$ and Abhishek Banerjee ${ }^{2}$ \\ ${ }^{1}$ Institute of Neuroscience, College of Natural Sciences, The University of Texas at Austin, Austin, Texas 78705, and ${ }^{2}$ Department of Brain and Cognitive \\ Sciences, The Picower Institute for Learning and Memory, Massachusetts Institute of Technology, Cambridge, Massachusetts 02139 \\ Review of Chen et al.
}

\begin{abstract}
A longstanding goal in neuroscience is to identify and understand synaptic mechanisms underlying the formation of neural circuits that perform sensory computations. Maturation of these circuits proceeds through developmental milestones like the critical period (CP), a time when sensory systems are patterned by afferent sensory activity. In primary visual cortex (V1), dramatic changes occur in the anatomical and functional organization of ocular preference during the $\mathrm{CP}$ when retinal inputs converge and compete (Katz and Crowley, 2002; Espinosa and Stryker, 2012). In V1 of mice and other mammals, neurons in the binocular zone (Fig. 1) shift preference toward the ipsilateral eye if the contralateral eye is occluded during the CP (Gordon and Stryker, 1996). Despite extensive exploration of experiencedependent mechanisms in mouse V1, the specific nature of synaptic competition shaping the formation of ocular preference in individual target neurons has remained unknown. Recently, Chen and colleagues (2014) shed light on this question by demonstrating that strong coinci-
\end{abstract}

Received April 18, 2014; revised May 18, 2014; accepted May 21, 2014.

B.S. is supported by NIH Training Grant T32EY021462. A.B. held a postdoctoral fellowship from the Simons Center for the Social Brain at MIT. We thank Drs. Andrew Tan and Samuel Cooke for critically reading the manuscript.

Correspondence should be addressed to Dr. Abhishek Banerjee, Picower Institute for Learning and Memory, Department of Brain and Cognitive Sciences, Massachusetts Institute of Technology, 43 Vassar Street, Cambridge, MA 02139. E-mail: abhii@mit.edu.

DOI:10.1523/JNEUROSCI.1598-14.2014

Copyright $\odot 2014$ the authors $\quad 0270-6474 / 14 / 348931-03 \$ 15.00 / 0$ dence of synaptic inputs from the two eyes is restricted to the CP. Their work suggests that temporal correlations of synaptic inputs between the two eyes, accompanied by an enhancement of GABAergic transmission, are essential features of the $\mathrm{CP}$ (Chen et al., 2014).

To reveal synaptic mechanisms of experience-dependent plasticity, Chen and colleagues (2014) performed in vivo whole-cell patch-clamp recordings from excitatory neurons in mouse $\mathrm{V} 1$, and measured subthreshold input conveying information from each eye before, during, and after the CP. Strikingly, they observed coincident subthreshold responses driven through the two eyes during the CP [postnatal day (P)25-P32]. Before or after the $\mathrm{CP}$, there was a temporal mismatch between responses driven through the two eyes. Before the CP (P17-P19), ipsilateral subthreshold responses took almost twice as long to arrive as contralateral ones. During the CP, monocular inputs had almost identical latencies, although ipsilateral responses showed greater variation across recordings. In adult animals $(\sim$ P 60$)$, binocular coincidence was abolished, with latency differences similar to those before CP onset. These measurements were made with sinusoidal gratings, but temporal coincidence during the CP was also shown using a sparse noise stimulus of bright pixels. These experiments demonstrate a previously unknown feature of the formation of binocular circuits during the $\mathrm{CP}$ of visual system development: temporal coincidence of synaptic inputs from each eye.

The authors next sought to understand the association between coincident binocular inputs and CP onset, which is thought to be regulated by maturation of inhibitory input onto cortical neurons (Hensch et al., 1998). To investigate this association, they induced a precocious CP by infusing diazepam (DZ), a positive modulator of $\mathrm{GABA}_{\mathrm{A}}$ receptor. Successful CP induction was demonstrated by monocular deprivation shifting ocular dominance of neurons away from contralaterally biased visual input. Surprisingly, DZ infusion before the CP induced binocular input coincidence, with subthreshold responses having similar latencies. Animals infused with only a drug vehicle showed response latencies typical of pre-CP observations. After dark rearing, which ablates the developmental CP, Chen and colleagues (2014) rescued the $\mathrm{CP}$ with DZ and again demonstrated binocular input coincidence, providing further evidence that CP onset via regulation of inhibition is accompanied by temporal coincidence of binocular inputs.

In a final experiment, the authors asked whether binocular coincidence is required for plasticity during the $\mathrm{CP}$. To answer this question, they tested whether desynchronizing ocular inputs during development was sufficient to impair ocular dominance plasticity. Using mice expressing channelrhodopsin-2 (a light-sensitive optogenetic actuator) in retinal ganglion 


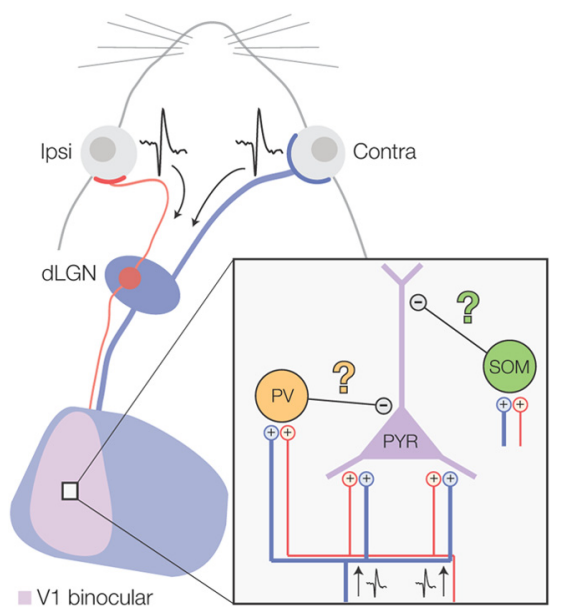

Figure 1. Binocular input integration in mouse visual cortex. Schematic diagram showing mouse visual system and stimulus presentation. Drifting gratings of different orientations and sparse noise stimuli were displayed on a screen in front of the mouse, while ipsilateral (Ipsi) and contralateral (Contra) eye responses were measured in vivo with whole-cell patch-clamp from principle neurons in the binocular region of primary visual cortex (V1 binocular). Diagram showing major neuronal subtypes in V1 [pyramidal (PYR), parvalbumin (PV), and somatostatin (SOM)] that may be differentially involved in processing eye-specific visual information.

cells allowed the authors to control retinal activity precisely through light stimulation. For 4 days preceding the $\mathrm{CP}$, the authors generated strong synchronous or asynchronous cortical activity through retinal channelrhodopsin stimulation. Effects of precise control on pre-CP visual experience were then tested during the $\mathrm{CP}$ with monocular deprivation under normal viewing without retinal light stimulation. Asynchronous stimulation negated both plasticity induced by monocular deprivation and binocular coincidence of synaptic input, while synchronous stimulation did not alter the normal CP or temporal coincidence of synaptic inputs. These results suggest that visual experience preceding the $\mathrm{CP}$ is essential for driving binocular coincidence, although it is perplexing why asynchronously driven retinal stimulation is dramatically different from ordinary pre-CP subthreshold inputs, since their temporal separations are comparable (60-70 ms). Perhaps more work is necessary to disambiguate differences between visual- and channelrhodopsinevoked cortical activity.

A natural question emerging from this study is whether coincidence or induction thereof is similar across different elements of V1 cortical circuitry. If coincidence is primarily driven by feedforward thalamocortical projections, this developmental change might occur primarily in thalamo- recipient layers (Hensch et al., 1998; Scholl et al., 2013b). Presumably both thalamocortical and intracortical inputs are necessary, since synchronous visual experience mediated through thalamocortical feedforward inputs and intracortical GABA transmission are essential for CP onset. Although Chen and colleagues (2014) found no laminar-specific differences among their reconstructed neurons, they did not examine differences between simple and complex cells. In general, V1 neurons with linear receptive fields receive more direct thalamocortical input (e.g., simple cells) than those with nonlinear properties (overlapping ON and OFF subregions, e.g., complex cells). By expanding on their sparse noise stimulus, using bright and dark pixels, the authors could have characterized receptive field linearity and input latency. This analysis could also help elucidate the relative contributions of thalamocortical and intracortical inputs in generating binocular coincidence. Furthermore, input-specific mechanisms with distinct spatiotemporal properties regulate the induction and expression of plasticity differentially in other brain regions (Banerjee et al., 2014), and these changes may occur at different cortical layers at different timescale.

How different cell types shape binocular coincidence was also unexplored in this study. Recordings of synaptic input latency differences between pyramidal cells, which are primarily excitatory, and interneurons, which are primarily inhibitory, would be highly informative (Fig. 1). Inhibition might directly shape binocular coincidence through gain modulation, shunting mechanisms, or by modulating inter-interneuronal connectivity (Pfeffer et al., 2013). A transient decrease in inhibition mediated by parvalbumin-expressing disinhibitory microcircuits has recently been linked to initiation of ocular dominance plasticity in mice during CP (Kuhlman et al., 2013). Interneurons could silence intracortical connections to enhance feedforward thalamocortical weights. This would require inhibition to be enhanced during the $\mathrm{CP}$ as the cortex awaits changes in ocular inputs, which is supported by Chen and colleagues' (2014) induction of a precocious CP with DZ. Knowledge of binocular coincidence progression immediately following enhancement of GABAergic transmission would help illuminate the idea. Disruption of the balance of synaptic excitation and inhibition could also be important for guiding binocular coincidence, as it is shown to underlie changes of binocular circuitry in strabismic amblyopia (Scholl et al., 2013b). However, whether similar neurodevelopmental disorders that feature gross alterations in early inhibition (Durand et al., 2012) also show defects in experience-dependent alignment and integration of functionally relevant synaptic inputs remains to be seen.

Discovery of binocular coincidence during the CP reveals a key mechanism underlying the formation of receptive fields and sensory processing in V1. Earlier studies (Wang et al., 2013) have demonstrated that receptive fields in neurons responding to the two eyes are unmatched before the CP, but align during the $\mathrm{CP}$ and persist thereafter. This matching could be mediated perfectly by coincident synaptic input, allowing plasticity to shape receptive field properties. Wang et al. (2013) also found that matching orientationselectivity for responses to both eyes requires visual experience, similar to that of binocular coincidence. An enticing experiment combining these two studies would be to alter early visual experience by restricting visual orientations during rearing. Placing orthogonal gratings on each eye would generate a mismatch, but may not affect binocular coincidence since certain structures like intermediate orientations would still provide some temporal coincidence. The presence of ocular input coincidence also strongly suggests that binocular responses of mouse V1 neurons are larger during the $\mathrm{CP}$, given simple linear or sublinear combination of inputs and assuming no change in synaptic weights. Chen and colleagues (2014) attempted to record binocular spiking responses during the $\mathrm{CP}$, but to accurately measure these responses the full range of binocular disparities must be explored using dichoptic stimulation, since these neurons are known to be sensitive to depth (Scholl et al., 2013a). Although it is unknown how depth sensitivity is shaped during the $\mathrm{CP}$, both binocular synaptic coincidence and receptive field matching are ideal mechanisms for the development of neuronal response properties.

What does binocular coincidence mean for this circuitry in generating behavior and the evolution of the early visual system? Despite only 3-4\% crossover of ipsilateral axons and $10 \%$ of the LGN output being ipsilateral (Scholl et al., 2013a), the binocular zone encompasses nearly a third of mouse V1. It appears the mouse visual system enhances and takes advantage of binocularity. In fact, these mechanisms and circuits might form the 
basis of an early, evolutionarily old binocular visual system that mice might use on a rudimentary level. It is also worth considering why temporal coincidence disappears after the CP. Perhaps mice rely mostly on switching between the visual fields of each eye, as opposed to convergent binocular viewing. Matching receptive field properties, potentially formed through binocular coincidence, might allow a seamless transition between representations (e.g., left to right monocular world). This type of visual exploration and interaction with the world has yet to be thoroughly studied in mice. As higher-order mammals had more retinal projections through thalamus to $\mathrm{V} 1$, and eyes became more forward-facing, creating larger binocular fields, these mechanisms and response properties became increasingly important and essential for their survival as a successful species.

\section{References}

Banerjee A, González-Rueda A, Baptista CS, Paulsen O, Rodríguez-Moreno A (2014) Distinct STDP induction rules at horizontal and vertical inputs on layer 2/3 neurons in mouse barrel cortex. Physiol Reports 2:e00271. CrossRef Medline

Chen XJ, Rasch MJ, Chen G, Ye CQ, Wu S, Zhang XH (2014) Binocular input coincidence mediates critical period plasticity in the mouse primary visual cortex. J Neurosci 34:29402955. CrossRef Medline

Durand S, Patrizi A, Quast KB, Hachigian L, Pavlyuk R, Saxena A, Carninci P, Hensch TK, Fagiolini M (2012) NMDA receptor regulation prevents regression of visual cortical function in the absence of Mecp2. Neuron 76:10781090. CrossRef Medline

Espinosa JS, Stryker MP (2012) Development and plasticity of the primary visual cortex. Neuron 75:230-249. CrossRef Medline

Gordon JA, Stryker MP (1996) Experiencedependent plasticity of binocular responses in the primary visual cortex of the mouse. J Neurosci 16:3274-3286. Medline

Hensch TK, Fagiolini M, Mataga N, Stryker MP, Baekkeskov S, Kash SF (1998) Local GABA circuit control of experience-dependent plas- ticity in developing visual cortex. Science 282: 1504-1508. CrossRef Medline

Katz LC, Crowley JC (2002) Development of cortical circuits: lessons from ocular dominance columns. Nat Rev Neurosci 3:34-42. CrossRef Medline

Kuhlman SJ, Olivas ND, Tring E, Ikrar T, Xu X, TrachtenbergJT (2013) A disinhibitory microcircuit initiates critical-period plasticity in the visual cortex. Nature 501:543-546. CrossRef Medline

Pfeffer CK, Xue M, He M, Huang ZJ, Scanziani M (2013) Inhibition of inhibition in visual cortex: the logic of connections between molecularly distinct interneurons. Nat Neurosci 16: 1068-1076. CrossRef Medline

Scholl B, Burge J, Priebe NJ (2013a) Binocular integration and disparity selectivity in mouse primary visual cortex. J Neurophysiol 109: 3013-3024. CrossRef Medline

Scholl B, Tan AY, Priebe NJ (2013b) Strabismus disrupts binocular synaptic integration in primary visual cortex. J Neurosci 33:1710817122. CrossRef Medline

Wang BS, Feng L, Liu M, Liu X, Cang J (2013) Environmental enrichment rescues binocular matching of orientation preference in mice that have a precocious critical period. Neuron 80:198-209. CrossRef Medline 